\title{
ESTRATÉGIAS EDUCATIVAS PARA PREVENÇÃO E REDUÇÃO DA MORBIMORTALIDADE DO CÂNCER DO COLO UTERINO
}

\section{Gilberto de Souza}

Mestrando em Biotecnologia pela Universidade Federal de São João del-Rei - UFSJ, Brasil.

E-mail: gilbertounifenas@yahoo.com.br

\section{Priscilla Santos Alves}

Bacharel em Enfermagem. Pós-Graduada em Educação e Saúde pela Universidade Federal de Minas Gerais - UFMG, Brasil.
RESUMO: Este estudo teve como objetivo analisar as estratégias educativas utilizadas pelo enfermeiro para a prevenção e redução de morbimortalidade do câncer de colo uterino no Brasil, buscando evidências na literatura sobre as estratégias educativas eficazes na prevenção do câncer de colo uterino. Para tanto foi realizada uma revisão integrativa da literatura. Esse estudo evidenciou a importância que a educação em saúde tem no contexto oncológico envolvendo o universo feminino. As ações de promoção da saúde são de extrema relevância, uma vez que envolvem a mulher no contexto da saúdedoença e enfocam o autocuidado. Neste estudo, foi possível constatar que as estratégias educativas utilizadas pelo enfermeiro para a prevenção de câncer do colo uterino são largamente diversificadas e necessitam ser combinadas entre si, pois, considerando-se que não há uma metodologia única e específica, elas devem ser adaptadas conforme as características loco-regionais do público alvo.

PALAVRAS-CHAVE: Educação em Saúde; Neoplasias do Colo do Útero; Prevenção de Câncer de Colo Uterino.

\section{EDUCATIONAL STRATEGIES FOR THE PREVENTION AND REDUCTION OF THE MORBIDIT Y-MORTALIT Y OF CERVICAL CANCER}

\begin{abstract}
Educational strategies employed by nurses for the prevention and reduction of morbidity-mortality rates of cervical cancer in Brazil are investigated. Educational strategies which are efficacious to prevent cervical cancer are retrieved from the literature. Current analysis demonstrated the important that health education has within the oncological context of the female. Health promotion activities are highly relevant since they involve women within the health-disease context and focus self-care. Collective educational strategies by nurses for the prevention of cervical cancer are diversified and required combination since there is no single and specific methodology. They should be adapted according to the local and regional characteristics of the target population.
\end{abstract}

KEY WORDS: Education in Health; Neoplasm of the Cervix; Prevention of Cervical Cancer

\section{INTRODUÇÃO}

O câncer (CA) do colo do útero é o terceiro tipo de câncer mais prevalente entre as mulheres no mundo todo, sendo também a 
quarta causa de morte feminina. As estimativas para o ano de 2015 mostram a possibilidade de ocorrer um aumento entre $14 \%$ a $16 \%$ na incidência e na mortalidade por esse tipo de câncer (SILVA et al., 2013).

No Brasil, o Instituto Nacional do Câncer (INCA) estima que o câncer de colo uterino seja a terceira neoplasia maligna mais comum entre as mulheres, onde é superado apenas pelo câncer de mama e do colorretal. Ainda segundo o INCA, no ano de 2014 foi esperada uma ocorrência de 15.590 novos casos de câncer do colo do útero, tendo-se como risco estimado 15,33 casos para cada 100 mil mulheres. É importante ressaltar que quando a doença é diagnosticada na fase inicial, as chances de cura são de 100\% (BRASIL, 2014).

A evolução do câncer de colo uterino, em geral, se dá de forma lenta, passando por fases pré-clínicas detectáveis e curáveis. Este é, dentre todos os tipos de câncer, o que apresenta um dos maiores potenciais de prevenção e cura. A faixa de idade para detecção precoce recomendada é entre 20 e 29 anos, sendo este o período que corresponde ao pico de incidência das lesões precursoras da doença e o período que antecede ao pico de mortalidade. O seu pico de incidência é encontrado nas mulheres com idade entre 40 e 49 anos de idade, onde apenas uma pequena porcentagem dos casos é encontrada nas mulheres com menos de 30 anos (CASARIN; PICCOLI, 2011).

Como fatores de risco, Lessa et al. (2012) e Uchimura et al. (2012) nos dizem que estão acometendo cada vez mais as mulheres em idade reprodutiva, sendo eles: exposição ao papiloma vírus humano (HPV), os antecedentes de doenças sexualmente transmissíveis (DST), o início precoce das relações sexuais, o tabagismo, o baixo nível socioeconômico e escolar, o uso de anticoncepcional oral, a multiparidade, além da multiplicidade de parceiros sexuais e o não uso do preservativo.

Dentre os vários fatores de risco para 0 desenvolvimento do câncer cérvico-uterino, a infecção pelo papiloma vírus humano (HPV) é apontado quase sempre como o agente etiológico desse tumor, sendo que $99 \%$ das neoplasias intraepiteliais e invasivas do trato genital feminino são causadas por um dos 15 tipos oncogênicos do HPV, onde os subtipos 16 e 18 são os mais prevalentes (LESSA et al., 2012).
Apesar disso, o câncer do colo do útero é passível de prevenção por meio de programas de rastreamento, que irão facilitar a detecção e o tratamento de lesões precursoras, tendo-se como objetivo a redução das taxas de incidência e de mortalidade da doença (SILVA et al., 2013). Havendo diagnóstico e tratamento precoce, é uma doença que apresenta um bom prognóstico. A detecção precoce é uma das ações de prevenção secundária, tendo como principal estratégia o rastreamento de mulheres sexualmente ativas por meio do exame citopatológico do colo uterino ou teste de Papanicolau (GASPERIN; BOING; KUPEK, 2011).

O exame de Papanicolau é, hoje, a principal estratégia para a detecção das lesões precursoras e para se fazer o diagnóstico da doença, uma vez que ele indica a presença de lesões neoplásicas ou pré-neoplásicas de células que são oriundas da ectocérvice e da endocérvice, extraídas por raspagem do colo do útero, possibilitando a interrupção da evolução das lesões. É um exame considerado eficiente e de baixo custo e vem sendo amplamente utilizado para o rastreamento do câncer de colo uterino na rede pública de saúde (SOARES; SILVA, 2010; CASARIN; PICCOLI, 2011; BRASIL, 2014).

A prevenção e a detecção precoce do câncer de colo uterino, além da coleta de exame de Papanicolau, devem envolver um conjunto de ações educativas, tendo como finalidade a divulgação das informações relevantes sobre o câncer e as suas formas de prevenção (SOARES; SILVA, 2010).

De acordo com estudos realizados por Marçal e Gomes (2013), a importância da educação em saúde dentro da comunidade está na quebra de barreiras e na desmistificação de crenças que atuam contrariamente às ações de promoção da saúde e de prevenção de agravos. Estes autores reforçam a importância de se realizar a busca ativa das mulheres, de se estabelecer vínculos com as mesmas, além de ressaltarem estratégias de educação em saúde e de intervenções em espaços comunitários, sendo estas ações realizadas dentro do planejamento de trabalho da Estratégia Saúde da Família, que se constitui em um importante aliado nas ações de prevenção do câncer de colo do útero.

Conforme resolução $n^{0}$ 381/2011 do Conselho Federal de Enfermagem (COFEN): 
[...] no âmbito da equipe de Enfermagem, a coleta de material para colpocitologia oncótica pelo método de Papanicolau é privativa do enfermeiro, observadas as disposições legais da profissão. O Enfermeiro deverá estar dotado dos conhecimentos, competências e habilidades que garantam rigor técnico-científico ao procedimento, atentando para a capacitação contínua necessária à sua realização.

A detecção precoce do câncer de colo de útero em mulheres assintomáticas, através do exame citopatológico, permite a detecção das lesões precursoras da doença nos seus estágios iniciais. Afim de se evitar o contágio pelo $\mathrm{HPV}$, a prevenção primária pode ser realizada através do uso de preservativos durante a relação sexual, uma vez que a prática de sexo seguro é uma das formas de evitar o contágio (CASARIN; PICCOLI, 2011).

A falta de adesão ao exame preventivo pela população feminina deve-se, em partes, a fatores como: desconhecimento do próprio corpo, desconhecimento do exame e de sua realização, dificuldade de acesso, demanda reprimida, falta de oportunidade que a usuária tem para falar sobre si e sobre sua sexualidade, como também, pelo desconhecimento sobre o próprio câncer, acrescido de sentimentos causadores de incômodo, medo e vergonha, tabus e ideias preconceituosas sobre a mulher (SOARES; SILVA, 2010; JORGE et al., 2011).

A falha no diagnóstico precoce pode ser explicada no conhecimento inadequado da população sobre a doença e na sua condição assintomática, bem como sobre a importância do exame preventivo e a possibilidade de tratamento precoce e o seu sucesso. Assim, é imprescindível que os profissionais de saúde que atuam diretamente na atenção primária busquem, além da eliminação dos fatores de risco, manifestar atitudes preventivas e de promoção da saúde, utilizando a educação em saúde e contribuindo para transformação social (OLIVEIRA et al., 2012).

O primeiro passo para a definição das estratégias de intervenção mais eficientes e adequadas consiste na compreensão de como uma determinada população percebe, sente e vive a saúde. No contexto do câncer do colo de útero, compreender os significados dos discursos das mulheres que realizaram o exame colpocitológico irá possibilitar o direcionamento do cuidar/assistir pela equipe de saúde, contribuindo para a transformação da prática assistencial e para melhorias na percepção das usuárias, proporcionando um atendimento mais adequado às necessidades da população feminina, além de um cuidar humanizado que, por sua vez, proporcionará maior adesão ao exame de Papanicolau (JORGE et al., 2011).

Assim, o conceito de educação em saúde irá associar-se ao conceito de promoção de saúde, uma vez que este está relacionado aos processos que necessitam da participação de toda a população, alcançando o contexto de sua vida cotidiana e tem como objetivo a capacitação desses indivíduos para a busca de melhoria das suas condições de saúde, visando a estimulação do diálogo, da reflexão e do saber (OLIVEIRA et al., 2012).

Assim sendo, as ações educativas que têm por objetivo promover mudanças de comportamento, devem ser fruto do diálogo e da participação, onde os profissionais de saúde, inclusive os enfermeiros, devem reconhecer as necessidades específicas de cada clientela, promovendo, assim, a saúde em todas as suas ações (LESSA et al., 2012).

O profissional enfermeiro exerce um papel fundamental, uma vez que dentre as suas atribuições, ele deve fazer uso dos espaços comunitários (escolas, empresas, salas de espera, etc.) para promover orientações, atendimentos individuais e grupos operacionais, realizando o denominado Processo de Enfermagem, de modo deliberado e sistemático. Assim, o enfermeiro se torna um profissional de fundamental relevância na prevenção de câncer de colo de útero no âmbito da atenção primária à saúde (BRASIL, 2009).

Mesmo diante de todas as possibilidades de tratamento precoce do câncer de colo de útero, ele é, ainda, um sério problema de saúde pública em países em desenvolvimento, apresentando altas taxas de prevalência e morbimortalidade em mulheres na fase produtiva de suas vidas, culminando com um prejuízo financeiro e social considerável, uma vez que essas mulheres, quando doentes, podem ocupar leitos hospitalares, ficando afastadas do mercado de trabalho e privadas do convívio familiar (RODRIGUES et al., 2012).

Neste contexto, percebe-se a importância do desenvolvimento de práticas educativas que visem a prevenção do câncer ginecológico, a sua detecção precoce, a promoção da saúde e a assistência ao tratamento, sendo 
de extrema relevância o papel realizado pelo enfermeiro no desenvolvimento das práticas educativas, objetivando tanto a saúde individual quanto a coletiva, promovendo uma transformação da realidade e estimulando a participação da comunidade nesse processo (OLIVEIRA et al., 2012).

Diante do exposto e levando-se em consideração que o câncer de colo uterino é uma neoplasia com elevada taxa de incidência e de morbimortalidade, passível de detecção precoce e de cura quando diagnosticado em seu início, o presente estudo tem como pergunta norteadora: Quais as estratégias educativas estão sendo utilizadas pelo enfermeiro para a prevenção e redução de morbimortalidade do câncer do colo uterino no Brasil?

Este trabalho foi motivado pelos questionamentos surgidos no dia a dia da prática de enfermagem na assistência à saúde da mulher, devido à baixa adesão das mesmas em realizar o exame de prevenção do câncer de colo do útero regularmente, expondo-as ao risco de desenvolver a doença. A escolha desse tema representou a necessidade de buscar mais informações em relação à promoção da saúde, através de uma análise de como são realizadas as atividades de prevenção de câncer do colo do útero.

Sendo assim, o presente estudo tem como objetivo analisar as estratégias educativas utilizadas pelo enfermeiro para a prevenção e redução de morbimortalidade do câncer de colo uterino no Brasil, buscando evidências na literatura sobre as estratégias educativas eficazes na prevenção do câncer de colo uterino.

Os autores desse trabalho entendem que é preciso conhecer as estratégias educativas utilizadas pelo enfermeiro, na prevenção e redução da morbimortalidade do câncer de colo uterino a fim de ampliar seus conhecimentos sobre o assunto e contribuir para a assistência na prática.

\section{METODOLOGIA}

Trata-se de um estudo originário de uma pesquisa bibliográfica, realizado através de um levantamento de publicações, procedendo-se a uma revisão integrativa da literatura. Optou-se pela revisão integrativa, que permite a inclusão de estudos de diferentes delineamentos de pesquisa, favorecendo o alcance do objetivo proposto, visto que é o mais amplo método referente às revisões, permitindo a inclusão de estudos experimentais e não experimentais para compreensão completa do fenômeno analisado (CAMELO, 2012).

Segundo Souza, Silva e Carvalho (2010), a revisão integrativa é um método que proporciona a síntese de conhecimento e a incorporação da aplicabilidade de resultados de estudos significativos na prática, onde seu desenvolvimento inclui as seguintes etapas: 1) elaboração da pergunta norteadora; 2) busca ou amostragem da literatura; 3) coleta de dados; 4) análise crítica dos estudos incluídos; 5) discussão dos resultados; 6) apresentação da revisão integrativa.

A revisão foi realizada a partir do levantamento e compilação de artigos por meio eletrônico, reunindo conhecimento sobre "Estratégias educativas utilizadas pelo enfermeiro, para prevenção e redução da morbimortalidade do câncer de colo uterino no Brasil", no intuito de produzir uma resposta para o problema abordado: "Quais estratégias educativas têm sido utilizadas pelo enfermeiro para a prevenção e redução da morbimortalidade do câncer do colo uterino no Brasil?".

A busca pelo material foi realizada nos meses de fevereiro e março de 2015 nas bases de dados da Biblioteca Virtual de Saúde, caracterizadas como Literatura Latinoamericana em Ciências da Saúde (LILACS), Base de dados Bibliográficos Especializada na Área de Enfermagem do Brasil (BDENF) e da biblioteca Medical Literature Analysis and Retrieval System Online (MEDLINE).

Foram utilizados os descritores controlados - DeCS (Descritores em Ciências da Saúde) na língua portuguesa e espanhola: "Neoplasias do colo do útero", "Prevenção de câncer de colo uterino" e "Educação em saúde".

Foi necessário, ainda, adotar critérios de inclusão relacionados a: artigos publicados no período de 10 anos, ou seja, de 2005 a 2014, em virtude da atualidade dos dados abrangidos por esta temporalidade, em periódicos nacionais e internacionais, que abordassem as estratégias educativas utilizadas pelo enfermeiro para prevenção e redução da mortalidade do câncer do colo uterino no Brasil e publicados nos idiomas português ou espanhol, os quais estivessem disponíveis na íntegra eletronicamente. 
Foram encontrados 486 artigos com o descritor Neoplasia do colo do útero, apenas dez atendiam aos critérios e após leitura dos resumos foram selecionados três. Com o descritor Prevenção de câncer de colo uterino encontrou-se 227, permanecendo sete após leitura dos títulos, sendo selecionados dois artigos que respondiam a questão norteadora. E com o descritor Educação em saúde foram identificados 4.162 trabalhos, sendo selecionados dois, perfazendo, ao todo, um total de sete artigos.

Realizou-se leitura do título e do resumo, sendo excluídos aqueles que não abordavam as questões norteadoras, os que estavam duplicados nas bases (sendo escolhidos em uma base e excluídos nas outras duas) e os que não forneciam acesso ao texto completo. Posteriormente, foi realizada leitura completa dos artigos e excluídos da amostra doze deles, por não abordarem especificamente a temática das estratégias educativas para a prevenção do câncer cérvico-uterino e, portanto, não respondiam a questão norteadora do estudo. Sendo assim, a amostra final foi composta por sete artigos.

Para a análise das informações, foi realizada a organização do material encontrado com identificação do periódico, ano de publicação, autoria, objetivo, delineamento, características do estudo e as estratégias utilizadas pelo enfermeiro para a prevenção de câncer do colo uterino.

A análise e discussão dos resultados abordam a utilização de estratégias educativas pelo enfermeiro para a prevenção do câncer do colo uterino. Nesse sentido, o estudo possibilitou o agrupamento dos dados em unidades temáticas relacionadas às estratégias educativas essenciais utilizadas pelo enfermeiro para a prevenção do câncer do colo uterino que serão apresentadas a seguir.

\section{RESULTADOS E DISCUSSÃO}

3.1 ESTRATÉGIAS UTILIZADAS PELOS ENFERMEIROS PARA A PREVENÇÃO E A REDUÇÃO DA MORBIMORTALIDADE DO CÂNCER DE COLO UTERINO

O enfermeiro que atua na Estratégia Saúde da Família (ESF) tem amplo foco de atuação na prevenção do câncer de colo do útero. $\mathrm{O}$ trabalho em equipe, embasado no planejamento das ações e na avaliação dos resultados alcançados auxilia na obtenção dos índices satisfatórios de promoção da saúde. O trabalho realizado em uma área adscrita específica, com população devidamente cadastrada e acompanhada pela ESF irá permitir que as estratégias propostas de educação em saúde e os trabalhos com a comunidade fortaleçam a participação social (MARÇAL; GOMES, 2013).

No contexto da prevenção do câncer do colo do útero é fundamental a atuação do enfermeiro nas equipes da ESF, onde suas atividades são desenvolvidas em diversas dimensões, a saber: realização das consultas de enfermagem, coleta do exame de Papanicolau, ações educativas junto à equipe de saúde e à comunidade, gerenciamento e contatos para o provimento de recursos materiais e técnicos, controle da qualidade dos exames realizados, verificação e comunicação dos resultados e encaminhamentos para os devidos procedimentos quando se fizer necessário (MELO et al., 2012).

No processo saúde-doença, a enfermagem desempenhaum papel importante, atuando nos diferentes níveis de prevenção, onde suas ações preventivas não são estáticas ou isoladas, formando um elo contínuo na história natural de qualquer distúrbio. Os programas e os serviços curativos têm a sua relevância, mas é fundamental procurar meios para reduzir a necessidade de sua utilização (PRADO; SILVEIRA, 2010).

Assim, faz-se necessária uma atuação diferenciada dos profissionais da saúde com as mulheres no que tange o exame de prevenção do câncer de colo do útero, em uma atuação com envolvimento, com respeito à intimidade e à privacidade da usuária, bem como ao seu direito de conhecer e de poder dialogar sobre o seu processo saúde-doença (JORGE et al., 2011).

Consideradas como portas de entrada do usuário no sistema de saúde, as Unidades de Atenção Primária à Saúde (UAPS) são espaços nos quais o enfermeiro é um importante integrante da equipe multiprofissional, exercendo atividades técnicas específicas de sua competência, administrativas e educativas, sendo que através do vínculo com as usuárias estão concentrados os esforços para reduzir os tabus, os mitos e os preconceitos e buscar o convencimento das usuárias sobre os benefícios da prevenção (MELO et al., 2012). 
Como formas para se convidar as usuárias para participar das ações, alguns autores citam a confecção e a distribuição de cartazes e panfletos. Sabe-se que a mídia impressa é uma estratégia limitada que, embora rica em imagens, não permite a interação entre os usuários e os serviços, sendo também um fator impeditivo para a adesão das usuárias analfabetas ou com dificuldades em entender a linguagem escrita. O material impresso possui baixo custo e permite ao usuário uma leitura posterior, melhorando o nível de conhecimento das mulheres em relação à realização do exame Papanicolau, porém, sem conseguir diminuir o nível de ansiedade em relação ao mesmo, sendo uma metodologia mais eficaz quando associada a outros métodos educativos (SILVA et al., 2012; RIQUELME; CONCHA; URRUTIA, 2012; RODRIGUES et al., 2012; VASCONCELOS et al., 2011).

Rodrigues et al. (2012) apresentam, como estratégia de comunicação, os programas de rádio, visto ser um dos meios de comunicação em massa mais acessíveis à população rural, tornando-o um importante canal para a realização de iniciativas que aproximam as diferentes realidades, com vistas ao crescimento cultural e educacional da população. Os programas de rádio, em geral, são de fácil entendimento, uma vez que se tratam de uma interlocução e uma interatividade entre o emissor e o receptor, apesar de apresentar limitações quanto à mensuração do alcance da mensagem enviada. Essa estratégia pode se tornar eficiente se houver um feedback durante a realização do programa ou se ocorrer aumento da demanda dos serviços de saúde explicitados na proposta.

Como outras estratégias passíveis de serem aplicadas nos serviços de saúde, destacam-se as conversas na sala de espera e sessões educativas com discussão em grupo. Estas atividades têm como impactos positivos, o estreitamento da relação entre as usuárias do serviço e o profissional da enfermagem, em virtude da interação entre ambos, permitindo a troca de saberes e a aprendizagem mútua. Para uma maior eficácia dessas intervenções é importante que as mesmas sejam combinadas com outras intervenções (PRADO; SILVEIRA, 2010; RODRIGUES et al., 2012; VASCONCELOS et al., 2012; RIQUELME; CONCHA; URRUTIA, 2012).

Uma outra proposta de ação educativa consiste na utilização de manequins, materiais demonstrativos e/ ou atividades lúdicas. Essas metodologias são maneiras de romper mitos e tabus em relação à coleta do material, permitindo à usuária conhecer os materiais a serem utilizados durante a coleta e a estrutura anatômica do corpo feminino, com seus estados fisiológicos e patológicos, desmitificando as inferências negativas que envolvem esse procedimento (OLIVEIRA et al., 2012; SILVA et al., 2012).

As intervenções lúdicas são consideradas importantes pois proporcionam uma mediação da aprendizagem, estimulando, de forma prazerosa, a compreensão do assunto, permitindo que haja uma reflexão sobre o conhecimento adquirido e favorecendo a formação de relações entre o conhecimento ofertado pela atividade lúdica e a realidade vivenciada, englobando os aspectos comportamentais individuais e coletivos (CROSCATO; PINA; MELLO, 2010).

Partindo do pressuposto que as pessoas somente precisariam de um estímulo (lembrete) para a prática da conduta adequada, as intervenções ditas como comportamentais, que utilizaram como estratégia lembretes, cartas ou telefonemas, são eficazes em relação à detecção precoce do câncer de colo uterino. Porém, elas remetem à necessidade de serem combinadas a outras práticas de intervenções para se alcançar uma melhor eficácia, devendo-se levar em consideração as características da população e a facilidade de acesso aos meios de comunicação. Outro ponto a ser considerado, refere-se à estrutura dos serviços de saúde, bem como a disponibilidade de profissionais e de recursos para a adoção dessa estratégia (VASCONCELOS et al., 2011).

As palestras, enquanto estratégia informativa, também apresentam impactos positivos na prevenção do câncer de colo uterino, uma vez que apresentam os sinais e os sintomas da doença, estimulando as usuárias a procurar pelos serviços de saúde e favorecendo a adesão ao exame preventivo e às ações de educação em saúde. Essa prática também permite ainda que as participantes possam demonstrar os seus conhecimentos sobre o procedimento, tanto em relação ao auto exame das mamas, quanto ao preventivo do câncer de colo de útero, além de destacar quais as suas finalidades e os locais que podem ser realizados (CASARIN; PICCOLI, 2011; PRADO; SILVEIRA, 2010; SILVA et al., 2012). 
As atividades que são realizadas em escolas de ensino médio, em praças públicas e feiras populares também são estratégias eficazes para a prevenção e o tratamento do câncer ginecológico e de mama, uma vez que têm maior abrangência e atingem um público diversificado, podendo ser utilizadas com outras estratégias, que incluem a distribuição de panfletos e atividades lúdicas, sendo recebidas de forma positiva pela população, incentivando as mulheres ao autoconhecimento (OLIVEIRA et al., 2012).

\subsection{AÇÕES EDUCATIVAS E SUA IMPORTÂNCIA PARA A PREVENÇÃO E A REDUÇÃO DA MORBIMORTALIDA- DE DO CÂNCER DE COLO UTERINO}

Visando a educação em saúde para a equipe e para a comunidade, as ações educativas realizadas na ESF e nas UBS abordam os temas de prevenção do câncer de colo de útero e importância de ser realizar o exame de Papanicolau, ressaltando a importância deste para a prevenção e o prognóstico quando diagnosticado precocemente. As atividades podem ser desenvolvidas em pequenos grupos, facilitando o diálogo e a troca de conhecimentos e experiências, onde é apresentado material demonstrativo, com realização de atividades lúdicas, proporcionando uma maior interação entre os membros do grupo (GASPERIN; BOING; KUPEK, 2011).

Atualmente, o conceito de educação em saúde está atrelado ao conceito de promoção de saúde, pois este é relacionado a processos que envolvem a participação da população no seu contexto cotidiano, tendo como objetivo a capacitação do indivíduo para a busca da melhoria das suas condições de saúde, estimulando o diálogo, a reflexão, a ação partilhada e o questionamento (OLIVEIRA et al., 2012).

As atividades educativas devem ser elaboradas e praticadas por todos os membros da equipe, uma vez que as usuárias possuem contato multiprofissional. É importante que os membros da equipe conheçam a realidade local e o perfil social e reprodutivo das usuárias, elaborando planos para atingir a situação apresentada. Além disso, quando as ações são realizadas por toda a equipe, não há sobrecarga de atividades para nenhum profissional (MELO et al., 2012).
As ações de ensino e saúde são importantes também para promover a adesão das pacientes ao exame preventivo, valorizando todas as etapas do processo e permitindo que o profissional de saúde sensibilize e conscientize o público alvo (PRADO; SILVEIRA, 2010).

Lima et al. (2012), discorrendo sobre o significado do ato de educar, nos diz o seguinte:

Educar não significa simplesmente
transmitir/adquirir conhecimentos.
Existe, no processo educativo, um
arcabouço de representações de
sociedade e de homem que se quer
formar. Através da educação as no-
vas gerações adquirem os valores
culturais e reproduzem ou transfor-
mam os códigos sociais de cada so-
ciedade. Assim, não há um processo
educativo asséptico de ideologias
dominantes, sendo necessária a
reflexão sobre o próprio sentido e
valor da educação na e para a socie-
dade.

Dessa maneira, as práticas educativas em saúde, referem-se tanto às atividades de educação em saúde, voltadas para o desenvolvimento de capacidades individuais e coletivas, com vistas à melhoria da qualidade de vida e da saúde, quanto às atividades de educação permanente, que são voltadas para os trabalhadores da área de saúde, sendo realizadas através da formação profissional contínua. Cabe ressaltar que as ações de saúde não implicam simplesmente a utilização de raciocínio clínico, de diagnóstico, de prescrição de cuidados e de avaliação da terapêutica instituída (BORGES et al., 2012).

Nesse contexto, percebe-se que a educação e a saúde são espaços de produção e de aplicação de saberes destinados ao desenvolvimento do ser humano, e o enfermeiro, embasado nesses conceitos e definições, deve buscar as práticas educativas em saúde para a prevenção de doenças e a promoção da qualidade de vida dos usuários (PRADO; SILVEIRA, 2010).

Apesar de estarem presentes em todos os níveis de atenção à saúde, as práticas educativas devem estar presentes em todos os níveis de atenção; é na atenção básica que as práticas educativas são mais desenvolvidas. Para que haja uma maior integração do conhecimento e uma maior resolutividade dos problemas apresentados, torna-se necessária uma integração de toda a equipe para 
a prática de atividades educativas, permitindo, assim, a multidisciplinaridade (BORGES et al., 2012).

Quando são desenvolvidas por todos os profissionais de saúde, as atividades educativas permitem que a abordagem seja explanada por meio de uma visão holística, integral e, portanto, completa. Assim, quando realizada por toda a equipe e respeitando as funções de cada profissional da saúde, ela ultrapassa os limites da assistência, desenvolvendo efetivamente a promoção e a prevenção da saúde (LIMA et al., 2012).

A educação em saúde é uma estratégia para a formação de comportamentos que promovem ou mantêm uma boa saúde, sendo uma prática social que contribui para a formação da consciência crítica dos indivíduos sobre os seus problemas de saúde, levando-se em consideração a sua realidade. Ela estimula a busca de soluções e a organização de ações individuais e coletivas e é considerada um recurso onde o conhecimento científico na área de saúde atinge o cotidiano das pessoas, pois a compreensão dos condicionantes de saúde-doença oferece subsídios para se adotar novos hábitos e novas condutas de saúde (RODRIGUES et al., 2012).

Apesar da inexistência de legislação que indique os profissionais de saúde como um imperativo para o desenvolvimento de ações educativas em saúde, estes, uma vez conhecedores dos saberes biomédicos, assumem o desafio de cuidar para a saúde. Acredita-se que eles estão mais preparados para realizá-la, não apenas por causa da sua visão holística do ser humano, mas por serem os profissionais que estão por mais tempo ao lado do paciente. $\mathrm{O}$ enfermeiro, em especial, se identifica com o papel de educador, entendendo a sua formação acadêmica como a garantia para exercer este papel com consciência (BORGES et al., 2012).

Apesar de todos os avanços tecnológicos, é importante considerar os recursos que têm as características da população para qual a proposta de intervenção está sendo preparada (por exemplo, o nível de escolaridade) e a viabilidade da aplicação da tecnologia a ponto de ser educado. Os grupos de discussão e folhetos informativos são as estratégias mais utilizadas, dado o seu baixo custo, tornando-se acessível e seguro à disseminação da informação necessária. Portanto, deve-se considerar que a sua utilização não seja a única metodologia utilizada, combinando estes, um com outro, ou com outros métodos, de acordo com os recursos disponíveis. O importante é utilizar um método que assegure a retenção do conteúdo entregue ao público alvo (RIQUELME; CONCHA; URRUTIA, 2012).

Historicamente, a enfermagem brasileira sempre esteve associada às questões de educação em saúde, visto que desde o início do século XX, com a criação da Escola de Enfermagem Ana Nery em 1923, houve a necessidade de formação de uma categoria profissional que fosse responsável pela educação sanitária da população (BORGES et al., 2012).

\section{CONSIDERAÇÕES FINAIS}

Esse estudo evidenciou a importância que a educação em saúde tem no contexto oncológico envolvendo o universo feminino. As ações de promoção da saúde são de extrema relevância, uma vez que envolvem a mulher no contexto da saúde-doença e enfoca o autocuidado. Os familiares e/ou os cuidadores desempenham um papel fundamental no que diz respeito ao incentivo e ao apoio a essas mulheres, tanto para as que buscam a prevenção quanto para as que já se encontram em tratamento.

Para que ocorra uma diminuição da morbimortalidade das mulheres e uma melhora na cobertura dos exames faz-se necessário um rastreamento daquelas usuárias que nunca realizaram o exame de Papanicolau ou que não o realizam conforme preconizado.

A preocupação com ações preventivas, nesse caso específico quanto ao câncer do colo do útero, vem ocorrendo em nível mundial. É importante ressaltar que essas ações preventivas trazem inúmeros benefícios para as usuárias, contribuindo também para os cofres públicos, visto que os gastos com tratamentos prolongados da doença serão diminuídos. Especificamente no Brasil, tem-se percebido um esforço representativo da política pública no sentido de melhorar a cobertura do rastreamento da doença, com grande investimento neste cenário, visando uma atenção de ótima qualidade para as usuárias. 
Neste contexto, a educação em saúde e a coleta do exame Papanicolau constituem um processo eficiente entre as diversas ações preventivas possíveis, servindo como um instrumento de transformação social, uma vez que promovem mudanças a partir da formulação de novos hábitos e atua diretamente na prevenção e na detecção precoce do câncer de colo do útero.

Dentre as ações de educação permanente (atividades educativas junto às usuárias, parcerias entre os serviços de saúde e as universidades e/ou escolas e organizações que trabalhem com esse tema), devem-se priorizar atividades educativas com vistas ao diagnóstico precoce e rastreamento em mulheres sintomáticas e assintomáticas, além de garantir o acesso aos métodos de diagnóstico e tratamento adequados.

Neste estudo, foi possível constatar que as estratégias educativas utilizadas pelo enfermeiro para a prevenção de câncer do colo uterino são largamente diversificadas e necessitam ser combinadas entre si, pois, considerando-se que não há uma metodologia única e específica, elas devem ser adaptadas conforme as características loco-regionais do público alvo. Essa reflexão contribui para a verificação das diferentes possibilidades de ações educativas e o processo de construção contínua do autoconhecimento, do diálogo e da reflexão, que irão culminar com a transformação da realidade e a participação da comunidade nesse processo.

Vale ressaltar, ainda, que este é um tema que necessita de aprofundamento e de outros estudos, no sentido de ampliar o conhecimento e as discussões acerca das estratégias educativas utilizadas na prevenção do câncer de colo uterino pelos profissionais de saúde, em especial, do enfermeiro.

\section{REFERÊNCIAS}

BORGES, M. C. L. A.; PONTE, K. M. A.; QUEIROZ, M. V. O.; RODRIGUES, D. P.; SILVA, L. M. S. da. Práticas educativas no ambiente hospitalar: reflexões sobre a atuação do enfermeiro. Rev Pesqui Cuid Fundam., Rio de Janeiro, v. 4, n. 3, p. 2592-97, jul./set., 2012.
BRASIL. Conselho Federal de Enfermagem. Resolução COFEN n $n^{0}$ 358/2009. Dispõe sobre a Sistematização da Assistência de Enfermagem e a implementação do Processo de Enfermagem em ambientes, públicos ou privados, em que ocorre o cuidado profissional de Enfermagem, e dá outras providências. Brasília, DF, 2009.

BRASIL. Conselho Federal de Enfermagem. Resolução COFEN $n^{0}$ 381/2011. Normatiza a execução, pelo Enfermeiro, da coleta de material para colpocitologia oncótica pelo método de Papanicolaou. Brasília, DF, 2011.

BRASIL. Ministério da Saúde. Instituto Nacional do Câncer - INCA. Estimativa 2014: incidência de câncer no Brasil. Rio de Janeiro: INCA, 2014.

CAMELO, S. H. H. Professional competences of nurse to work in Intensive Care Units: an integrative review. Rev Latino-Am. Enfermagem, Ribeirão Preto, v. 20, jan./fev. 2012.

CASARIN, M. R.; PICCOLI, J. C. E. Educação em saúde para prevenção do câncer de colo do útero em mulheres do município de Santo Ângelo/RS. Ciênc Saúde Coletiva, v. 16, n. 9, p. 3925-3932, 2011.

CROSCATO, G; PINA, J. C.; MELLO, D. F. Utilização de atividades lúdicas na educação em saúde: uma revisão integrativa da literatura. Acta Paul Enferm., Ribeiro Preto, v. 23, n. 2, p. 257-63, 2010.

GASPARIN, S. I.; BOING, A. F.; KUPEK, E. Cobertura e fatores associados à realização do exame de detecção do câncer de colo de útero em área urbana no Sul do Brasil: estudo de base populacional. Cad Saúde Pública, Rio de Janeiro, v. 27, n. 7, July, 2011.

JORGE, R. J. B.; DIOGENES, M. A. R.; MENDONÇA, F. A. C.; SAMPAIO, L. R. L.; JORGE-JUNIOR, R. Exame Papanicolau: sentimentos relatados por profissionais de enfermagem ao se submeterem a esse exame. Ciênc Saúde Coletiva, Rio de Janeiro, v. 16, n. 5, May, 2011.

LESSA, P. R. A.; RIBEIRO, S. G.; LIMA, D. J. M.; NICOLAU, 
A. I. O.; DAMASCENO, A. K. C.; PINHEIRO, A. K. B. Presença de lesões intraepiteliais de alto grau entre mulheres privadas de liberdade: estudo documental. Rev Latino-Am. Enfermagem, Ribeirão Preto, v. 20, n. 2, mar./abr., 2012.

LIMA, M. O. M.; CARNEIRO, C. A.; RAMOS, G. G.; SANTOS, E. M. R.; PIVA, S. G. N. Implantação de salas educativas na estratégia de saúde da família por meio do agente comunitário de saúde como educador em saúde: um relato de experiência. Rev Bras Tecnol Sociais, Itajaí, v. 1, n. 1, p. 17-24, 2012.

MARÇAL, J. A.; GOMES, L. T. S. A prevenção do câncer de colo de útero realizada pelo enfermeiro na Estratégia Saúde da Família: revisão integrativa da literatura. REAS/ EJCH, Campinas, v. 5, n. 2, p. 474-489, 2013.

MELO, M. C. S.; VILELA, F.; SALIMENA, A. M. O.; SOUZA, I. E. O. O enfermeiro na prevenção do câncer do colo do útero: o cotidiano da atenção primária. Rev Bras Cancerol., Rio de Janeiro, v. 58, n. 3, p. 389-398, 2012.

OLIVEIRA, A. M.; POZER, M. Z.; SILVA, T. A.; PARREIRA, B. D. M.; SILVA, S. R. Ações extensionistas para a prevenção e o tratamento do câncer ginecológico e de mama: relato de experiência. Rev Esc Enferm. USP, São Paulo, v. 46, n. 1, Feb, 2012.

PRADO, M. R. M. C.; SILVEIRA, C. L. P. Atenção primária à saúde da mulher: um enfoque educativo-preventivo no combate ao câncer de colo de útero. Rev Enferm UFPE online, Recife, v. 4, n. 3, p. 1417-425, jul./set, 2010.

RIQUELME, G.; CONCHA, X.; URRUTIA, M. T. Intervenciones educativas para laprevencióndel câncer Cervicouterino. Rev Chil Obstet Ginecol, Santiago, v. 77, n. 2, p. 111-115, 2012.

RODRIGUES, B. C.; CARNEIRO, A. C. M. O.; SILVA, T. L.; SOLÁ, A. C. N.; MANZI, N. M.; SCHECHTMAN, N. P.; MAGALHÃES, H. L. G. O.; DYTZ, J. L. G. Educação em saúde para a prevenção do câncer cérvico-uterino. RBEM, Rio de Janeiro, v. 36, n. 1, p. 149-154, 2012
SILVA, M. G. P.; ALMEIDA, R. T.; BASTOS, E. A.; NOBRE, F. F. Determinantes da detecção de atipias celulares no programa de rastreamento do câncer do colo do útero no Rio de Janeiro, Brasil. Rev Panam Salud Pública, Washington, v. 34, n. 2, p. 107-113, aug. 2013.

SILVA, S. R.; LICIO, F. C.; BORGES, L. V.; MENDES, L. C.; VICENTE, N. G.; GOMES, N. S. Atividades educativas na área da saúde da mulher: um relato de experiência. Rev Enferm atenção saúde [Online], Uberaba, v. 1, n. 1, p. 106-112, 2012.

SOARES, M. B. O.; SILVA, S. R. Análise de um programa municipal de prevenção do câncer cérvico-uterino. REBEn, Brasília, v. 63, n. 2, p. 177-182, mar./apr. 2010.

SOUZA, M. T.; SILVA, M. D.; CARVALHO, R. Revisão Integrativa: o que é e como fazer. Rev Einstein, São Paulo, v. 6, p. 102-106, jun., 2010.

UCHIMURA, N.S.; UCHIMURA, T.T.; MARTINS, J.P.O.B.; ASSAKAWA, F.; UCHIMURA, L.Y.T. Avaliação da conduta conservadora na lesão intraepitelial cervical de alto grau. Rev Saúde Pública, São Paulo, v. 46, n. 3, p. 466-471, Jun., 2012.

VASCONCELOS, C. T. M.; DAMASCENO, M. M. C.; LIMA, F. E. T.; PINHEIRO, A. K. B. Revisão integrativa das intervenções de enfermagem utilizadas para deteç̧ão precoce do câncer cérvico-uterino. Rev Latino-Am. Enfermagem, Ribeirão Preto, v. 19, n. 2, p. 01-08, mar./ abr., 2011.

Recebido em: 29 de junbo de 2015 Aceito em: 27 de agosto de 2015 